\title{
Vertebral bone marrow T2* mapping using chemical shift encoding-based water-fat separation in the quantitative analysis of lumbar osteoporosis and osteoporotic fractures
}

\author{
Yannik Leonhardt ${ }^{1}$, Florian T. Gassert ${ }^{1}$, Georg Feuerriegel ${ }^{1}$, Felix G. Gassert ${ }^{1}$, Sophia Kronthaler ${ }^{1}$, \\ Christof Boehm ${ }^{1}$, Alexander Kufner ${ }^{1}$, Stefan Ruschke ${ }^{1}$, Thomas Baum ${ }^{2}$, Benedikt J. Schwaiger ${ }^{2}$, \\ Marcus R. Makowski ${ }^{1}$, Dimitrios C. Karampinos ${ }^{1}$, Alexandra S. Gersing ${ }^{1,3}$ \\ ${ }^{1}$ Department of Radiology, Klinikum Rechts der Isar, School of Medicine, Technical University of Munich, Munich, Germany; ${ }^{2}$ Department of \\ Neuroradiology, Klinikum Rechts der Isar, School of Medicine, Technical University of Munich, Munich, Germany; ${ }^{3}$ Department of Neuroradiology, \\ University Hospital of Munich (LMU), Munich, Germany
}

Contributions: (I) Conception and design: AS Gersing, T Baum, DC Karampinos, Y Leonhardt; (II) Administrative support: MR Makowski, AS Gersing, DC Karampinos, T Baum; (III) Provision of study materials or patients: DC Karampinos, T Baum, C Boehm, S Kronthaler, S Ruschke, MR Makowski; (IV) Collection and assembly of data: Florian T. Gassert, Felix G. Gassert, C Boehm, S Kronthaler, G Feuerriegel, A Kufner, Y Leonhardt; (V) Data analysis and interpretation: Y Leonhardt, AS Gersing, BJ Schwaiger, C Boehm, S Kronthaler, T Baum, DC Karampinos; (VI) Manuscript writing: All authors; (VII) Final approval of manuscript: All authors.

Correspondence to: Yannik Leonhardt. Department of Radiology, Klinikum Rechts der Isar, School of Medicine, Technical University of Munich, Ismaninger Strasse 22, 81675 Munich, Germany. Email: yannik.leonhardt@tum.de.

Background: Chemical shift encoding-based water-fat separation techniques have been used for fat quantification [proton density fat fraction (PDFF)], but they also enable the assessment of bone marrow T2*, which has previously been reported to be a potential biomarker for osteoporosis and may give insight into the cause of vertebral fractures (i.e., osteoporotic $v s$. traumatic) and the microstructure of the bone when applied to vertebral bone marrow.

Methods: The 32 patients $(78.1 \%$ with low-energy osteopenic/osteoporotic fractures, mean age $72.3 \pm 9.8$ years, $76 \%$ women; $21.9 \%$ with high-energy traumatic fractures, $47.3 \pm 12.8$ years, no women) were frequency-matched for age and sex to subjects without vertebral fractures $(n=20)$. All study patients underwent 3T-MRI of the lumbar spine including sagittally acquired spoiled gradient echo sequences for chemical shift encoding-based water-fat separation, from which T2* values were obtained. Volumetric trabecular bone mineral density (BMD) and trabecular bone parameters describing the three-dimensional structural integrity of trabecular bone were derived from quantitative CT. Associations between T2* measurements, fracture status and trabecular bone parameters were assessed using multivariable linear regression models.

Results: Mean T2* values of non fractured vertebrae in all patients showed a significant correlation with BMD ( $\mathrm{r}=-0.65, \mathrm{P}<0.001)$, trabecular number $(\mathrm{TbN})(\mathrm{r}=-0.56, \mathrm{P}<0.001)$ and trabecular spacing (TbSp) (r=0.61, $\mathrm{P}<0.001)$; patients with low-energy osteoporotic vertebral fractures showed significantly higher mean $\mathrm{T}^{*}$ values than those with traumatic fractures $(13.6 \pm 4.3 \mathrm{vs} .8 .4 \pm 2.2 \mathrm{~ms}, \mathrm{P}=0.01)$ as well as a significantly lower TbN $(0.69 \pm 0.08$ vs. $0.93 \pm 0.03 \mathrm{~mm}-1, \mathrm{P}<0.01)$ and a significantly larger trabecular spacing (1.06 \pm 0.16 vs. $0.56 \pm 0.08 \mathrm{~mm}, \mathrm{P}<0.01)$. Mean $\mathrm{T} 2 *$ values of osteoporotic patients with and without vertebral fracture showed no significant difference $(13.5 \pm 3.4$ vs. $15.6 \pm 3.5 \mathrm{~ms}, \mathrm{P}=0.40)$. When comparing the mean $\mathrm{T}^{*}$ of the fractured vertebrae, no significant difference could be detected between low-energy osteoporotic fractures and high-energy traumatic fractures $(12.6 \pm 5.4$ vs. $8.1 \pm 2.4 \mathrm{~ms}, \mathrm{P}=0.10)$.

Conclusions: $\mathrm{T}^{*}$ mapping of vertebral bone marrow using using chemical shift encoding-based water-fat separation allows for assessing osteoporosis as well as the trabecular microstructure and enables a radiation- 
free differentiation between patients with low-energy osteoporotic and high-energy traumatic vertebral fractures, suggesting its potential as a biomarker for bone fragility.

Keywords: Osteoporosis; magnetic resonance imaging (MRI); spine; bone density; T2* mapping; chemical shift encoding-based water-fat separation

Submitted Dec 19, 2020. Accepted for publication Apr 07, 2021. doi: 10.21037/qims-20-1373

View this article at: http://dx.doi.org/10.21037/qims-20-1373

\section{Introduction}

Osteoporosis is a metabolic bone disease characterised by an overall reduced bone mass that results in reduced bone strength $(1,2)$. The prevalence of osteoporosis and its consequences, e.g., low-energy vertebral fractures (VF), is continually increasing due to an overall ageing population (3). Complications such as pain and immobilisation are a burden for the individual patient as well as for the health care and social security systems, as they are confronted with increasing subsequent financial costs (4). Early detection and assessment of the osteoporotic status is of high importance, since it is a health condition that can potentially be treated, reducing the risk for osteoporotic fractures, pain and immobilisation (5).

Dual-energy X-ray absorptiometry (DXA) and dedicated quantitative CT (QCT) are the clinical standards for assessing osteoporosis by determining the bone mineral density (BMD) (4). However, those methods require additional radiation exposure and costs. Lack of access to dedicated diagnostic tools for BMD measurement and underutilization (e.g., due to patient noncompliance) potentially result in a multitude of missed diagnoses of osteoporosis despite the presence of a fragility fracture. This diagnostic gap leads to a delay of adequate care; previous studies have shown that in patients who sustain a fragility fracture, fewer than $20 \%$ of individuals receive therapies to reduce the risk of future fractures within the year following the fracture (6).

In clinical routine, the majority of the patients with suspected vertebral fracture receive imaging of the spine, e.g., in the form of radiography or CT for fracture detection and characterization as well as magnetic resonance imaging (MRI) for determination of the fracture age and the assessment of discoligamentous structures (7).

Previous studies have reported on $\mathrm{T} 2 *$ mapping and high-resolution trabecular bone imaging using MRI for the assessment of bone microstructure and osteoporosis
$(8,9)$. As bone is more diamagnetic than marrow, the trabecular bone and bone marrow interface induce local magnetic field inhomogeneities, which can be measured as a shortening of the effective transverse relaxation time (10). $\mathrm{T}^{*}$ measurements of the vertebra correlated with the bone mineral density inversly in previous studies, suggesting decreased susceptibility due to remodeled and decreased trabecular bone (11).

It has been shown that measurement of the fat content of the bone marrow itself is a reliable biomarker for quantification of osteoporosis, as the vertebral bone marrow composition changes, consisting of a mixture of hematopoetic tissue and adipocytes interspersed within the network of trabecular bone matrix $(12,13)$. Chemical shift encoding-based water-fat separation techniques have been used for fat quantification [proton density fat fraction (PDFF)] and assessment of osteoporosis, but they also enable the assessment of bone marrow $\mathrm{T} 2{ }^{*}$ in red marrow regions (14-16). $\mathrm{T} 2{ }^{*}$ assessed using chemical shift encoding-based water-fat imaging (CSEMRI) has previously been reported to be a biomarker for osteoporosis (14). It may give insight into the cause of vertebral fractures (i.e., osteoporotic vs. traumatic vs. malignant) when applied to vertebral bone marrow. In a more recent study, Schmeel et al. have already investigated the potential of $\mathrm{T}^{*}$ measurements to differentiate between acute benign and malignant vertebral fractures (17). To our knowledge however, it remains unknown whether T2* mapping using CSE-MRI enables the further differentiation between patients with high-energy traumatic and low-energy osteoporotic/osteopenic fractures as well as osteoporotic/osteopenic patients with and without vertebral fractures.

Therefore, the purpose of this study was to evaluate $\mathrm{T} 2 *$ mapping in vertebral bone marrow using chemical shift encoding-based water-fat separation in patients with and without osteoporotic and traumatic vertebral fractures and to assess their association with the trabecular bone 
microstructure.

\section{Methods}

\section{Patient selection and study design}

From January 2018 until July 2019 patients with a suspected vertebral fracture who underwent a CT examination at our institution as part of the clinical routine diagnostic work-up were approached for this study.

Patients with a CT examination as primary imaging modality, from which a quantitative BMD measurement could be derived, and with the diagnosis of at least one VCF were included in this study. Exclusion criteria were contraindications for MR imaging (e.g., pacemaker or pregnancy), history of cancer or hematologic disorders and withdrawal of consent.

In the initial assessment of the fractures we evaluated the clinical information and classified the fractures into lowenergy osteoporotic/osteopenic fractures in patients with acute low-energy trauma (e.g., falling from standing height or less) or sudden onset of backpain without any trauma and in acute high-energy fractures with an underlying adequate trauma (any other type of trauma, e.g., falling from height higher than standing and motor vehicle accident) (18). In total, 32 patients with vertebral fractures [25 (78.1\%) patients with in total 31 low-energy osteopenic/osteoporotic fractures, mean age $72.3 \pm 9.8$ years, $76 \%$ female; $7(21.9 \%)$ patients with in total 7 high-energy fractures, $47.3 \pm 12.8$ years, $0 \%$ female] agreed to participate in this study and gave written and informed consent. Out of the 32 patients 6 had two VCFs and 26 had one VCF. Patients with vertebral fractures were frequency-matched for age and sex to patients without vertebral fractures $(n=20,69.3 \pm 10.1$ years, 14 women).

The study was conducted in accordance with the Declaration of Helsinki (as revised in 2013). The study was approved by the local institutional review board (Ethics Commission of the Medical Faculty, Technical University of Munich, Germany; Ethics proposal number 70/17S). Written informed consent was obtained from all study participants.

\section{QCT and BMD measurements}

CT images were acquired with one dual-layer dualenergy (DLDE-) CT scanner (IQon Spectral CT, Philips Healthcare) and one multislice detector (MD-) CT scanner
(iCT 256, Philips Healthcare). The data was acquired in helical mode with a peak tube voltage of $120 \mathrm{kVp}$, a reconstruction slice thickness of 0.9 to $1 \mathrm{~mm}$ and adaptive tube load.

For BMD measurements, a mid-line $15 \mathrm{~mm}$ multiplanar reconstruction section in sagittal reformations was created with a PACS tool (IDS7, Sectra). Then, cylindrical volumes of interest were manually positioned in all non-fractured lumbar vertebrae by one radiologist (FTG), and mean Hounsfield Units (HU) were noted (19). Fractured or otherwise altered vertebrae (e.g., vertebrae with severe degenerative changes, vertebrae after vertebro-/kyphoplasty) were not used for $\mathrm{HU}$ measurements. The $\mathrm{HU}$ values were then converted into BMD using conversion equations as previously described: (I) $0.928 \times \mathrm{HU}+4.5 \mathrm{mg} / \mathrm{cm}^{3}$ for the IQon Spectral CT, (II) $0.855 \mathrm{~g} \times \mathrm{HU}+1.172 \mathrm{mg} / \mathrm{cm}^{3}$ for the Philips iCT 256 (20,21). Osteoporosis was defined as BMD $<80 \mathrm{mg} / \mathrm{cm}^{3}$ and osteopenia as $80 \leq \mathrm{BMD} \leq 120 \mathrm{mg} / \mathrm{cm}^{3}$ (22).

\section{MRI and T2* assessment}

Two 3T-MRI systems (one Ingenia, Philips Healthcare and one Elition, Philips Healthcare) were used for the dedicated examination of the lumbar spine. A six-echo 3D monopolar time-interleaved multi-echo spoiled gradient-echo sequence was acquired in sagittal orientation (23) using the posterior coil elements integrated in the table (12-channel array).

The six echoes were acquired in 2 interleaves with 3 echoes per repetition time $(\mathrm{TR})$ and $\mathrm{TR} / \mathrm{TE} 1 / \Delta \mathrm{TE}=8.2 / 1.3 / 1.1 \mathrm{~ms}$, field of view $(\mathrm{FOV})=626 \times 511 \times 102 \mathrm{~mm}^{3}$, voxel size $=1.8 \mathrm{~mm}^{3}$ isotropic, receiver bandwidth $=1,504 \mathrm{~Hz} /$ pixel, frequency direction=anterior/posterior $(\mathrm{A} / \mathrm{P}), 1$ average, scan time $=3 \min 39 \mathrm{~s}$. To minimize T1-bias effects a flip angle of $3^{\circ}$ was used (24). Frequency encoding was performed in $\mathrm{A} / \mathrm{P}$ direction to reduce respiratory motion artifacts. For the reconstruction, an image reconstruction toolbox from raw k-space data was used (ReconFrame, Gyrotools, Switzerland). The oversampling was not removed, yielding the above extended FOV. The $\mathrm{T} 2{ }^{*}$ maps were calculated using chemical shift encoding-based water-fat separation performed offline. A signal model accounting for the multi-peak fat spectrum and assuming a single $\mathrm{T}^{*}$ decay was employed (25). A multi-peak fat model specific to bone marrow with 9 fat peaks was used as previously introduced by Ren et al. (26). A state-of-the-art graph-cut algorithm (27) was used for fieldmap estimation and the parameter estimation was refined with a recently proposed generalized method for parameter estimation in multi-echo gradient-echo experiments (28). 

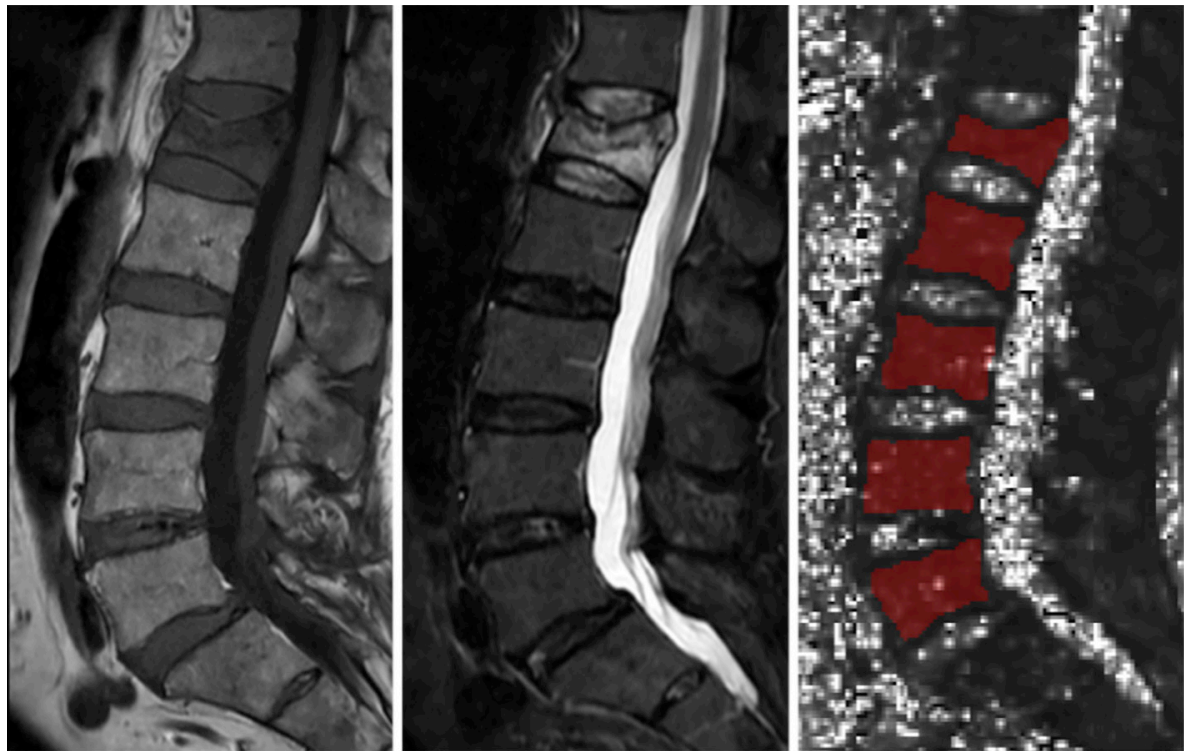

Figure $1 \mathrm{~T} 1 \mathrm{w}$ spin-echo sequence, STIR sequence and T2* map of the lumbar spine in sagittal orientation of a 73-year-old male patient with a VCF of L1. The manually segmented ROIs in the vertebral bodies L1 to L5 are depicted in red. VCF, vertebral compression fracture; ROI, region of interest; STIR, short-tau inversion recovery.

Furthermore, the MR imaging protocol encompasses a sagittal short-tau inversion recovery (STIR) as well as a T1w spin-echo sequence according to clinical standards.

The entire vertebral bodies Th12 to L5 were manually segmentated by a radiologist (F.T.G., with 5 years of experience in MSK imaging) using the open-source software Medical Imaging Interaction Toolkit (MITK, German Cancer Research Center, Heidelberg, Germany). Heavily altered vertebrae (e.g., after kyphoplasty, heavy degenerative alterations) were excluded. The mean $\mathrm{T} 2$ * value was calculated for each vertebra (fractured and non fractured) (Figure 1), then an average value was calculated for the non fractured vertebrae in each patient. In order to assess the intra-reader reproducibility error of the $\mathrm{T}^{*}$ and $\mathrm{BMD}$ values, a random sample of 5 subjects was chosen and reanalyzed by the same radiologist.

\section{Analysis of the MR imaging}

The morphologic imaging features of the vertebral fractures were assessed by two board-certified radiologists (BJS. and ASG, with 11 years and 9 years of experience in musculoskeletal imaging). In total, 47 vertebral fractures were identified. The radiologists also ensured, in context with the clinical history, that no morphologic signs for a malignant fracture were present in any of the sequences.

\section{CT texture analysis of the trabecular bone}

Trabecular bone analysis was performed using the $C A L C D C N$ software developed at the University of California, San Francisco, which is based on IDL (Interactive Display Language, Research Systems, Inc., CO, USA).

Axial images of the spine (reconstructed in the bone kernel) were binarized by absolute thresholding to bone ("on" pixels) and marrow ("off" pixels). A global threshold was applied to all images, which was optimized visually prior to all analysis procedures as previously described by other researchers $(29,30)$. The analyzed parameters included the trabecular number $\left[\mathrm{TbN}\left(\mathrm{mm}^{-1}\right)\right]$, spacing $[\mathrm{TbSp}(\mathrm{mm})]$ and thickness [TbTh (mm)].

\section{Statistical analysis}

Statistical analysis was performed with SPSS version 25 software (IBM). Differences in baseline characteristics between osteoporotic patients with and without fractures or between patients with osteoporotic and traumatic fractures were assessed using independent sampes t-tests for continuous variables and chi-square tests for categorical variables. Pearson Correlation Coefficient was used to assess the correlations between $\mathrm{T} 2^{*}$ values, trabecular parameters and BMD. Differences in $\mathrm{T}^{*}$ values between patients 
Table 1 Characteristics of patients with and without at least one vertebral fracture

\begin{tabular}{|c|c|c|c|c|}
\hline Characteristics & With fracture $(n=32)$ & Without fracture $(n=20)$ & All $(n=52)$ & $P$ value \\
\hline Women & 20 & 14 & 34 & \\
\hline Men & 12 & 6 & 18 & \\
\hline Age (years) & $66.4 \pm 14.4$ & $69.3 \pm 10.1$ & $67.5 \pm 12.9$ & 0.44 \\
\hline Normal $\left(\geq 120 \mathrm{mg} / \mathrm{cm}^{3}\right)$ & 7 & 13 & 20 & \\
\hline Reduced $\left(<120 \mathrm{mg} / \mathrm{cm}^{3}\right)$ & 25 & 7 & 32 & \\
\hline
\end{tabular}

$\mathrm{BMD}$, bone mineral density; QCT, quantitative computed tomography.

with and without vertebral fractures in the osteoporotic/ osteopenic group and differences between patients with osteoporotic/osteopenic fractures and traumatic fractures were calculated using a multivariable linear regression model, adjusting for age, sex and BMD. Significance was assumed if $\alpha$-level was $\mathrm{P}<0.05$.

For the measurement of the intra-rater reproducibility error of the $\mathrm{T}^{*}$ and $\mathrm{BMD}$ values, the root mean square errors (RMSE) were calculated in order to assess the differences between the respective measurements.

\section{Results}

In this study, 52 patients in total were included $(65 \%$ female, $67.5 \pm 12.9$ years). In total, 32 patients had at least one vertebral fracture $(78.1 \%$ with low-energy osteopenic/ osteoporotic fractures, mean age $72.3 \pm 9.8$ years, $76 \%$ female; $21.9 \%$ with high-energy fractures, $47.3 \pm 12.8$ years, $0 \%$ female); out of the 32 patients 6 had two VCFs and 26 had one VCF. According to the QCT-based BMD measurements, a total number of 32 patients were classified as osteoporotic/ osteopenic $(32 / 52 ; 62.0 \%)$, in accordance with the abovestated definition of osteoporosis/osteopenia. When analyzing the data of all patients, between patients with and without vertebral fractures, there were no significant differences found in age $(66.4 \pm 14.4$ vs. $69.3 \pm 10.1$ years respectively; $\mathrm{P}=0.44)$ and sex $[\mathrm{n}=20$ female patients $(62.5 \%)$ vs. $\mathrm{n}=14$ (70.0\%), respectively, $\mathrm{P}=0.59$ ] (Table 1 ).

When assessing $\mathrm{T} 2$ * of non fractured vertebrae in all patients, the mean $\mathrm{T} 2{ }^{*}$ values showed an inverse correlation with the BMD values (Pearson's $\mathrm{r}=-0.65, \mathrm{P}<0.001$ ). $\mathrm{T} 2^{*}$-values were significantly higher in patients with osteoporosis/osteopenia compared to patients with normal $\mathrm{BMD}(13.6 \pm 4.2$ vs. $9.3 \pm 2.5 \mathrm{~ms}, \mathrm{P}=0.006)$. Moreover, $\mathrm{T} 2$ * values also showed a moderate to high correlation with the trabecular number and trabecular spacing $(r=-0.56$, $\mathrm{P}<0.001$ and $\mathrm{r}=0.61, \mathrm{P}<0.001$, respectively). As expected, the correlation between the microstructure parameters trabecular number $(\mathrm{TbN})$, trabecular spacing $(\mathrm{TbSp})$ and the BMD were high $(r=0.71$ and $r=-0.69, P<0.001$ for both correlations, respectively). Furthermore, with older age $\mathrm{T} 2 *$-values increased significantly $(\mathrm{r}=0.70, \mathrm{P}<0.001)$ while $\mathrm{BMD}$-values decreased significantly $(\mathrm{r}=-0.63, \mathrm{P}<0.001)$ (Table 2 and Figure 2). No significant differences between $\mathrm{T} 2{ }^{*}$-values of the vertebrae between women and men were found (12.9 4 4.2 vs. $10.8 \pm 3.7 \mathrm{~ms}, \mathrm{P}=0.17)$.

Out of all 52 patients 32 had at least one vertebral fracture, whereas 25 were classified as osteoporotic/ osteopenic $(25 / 32 ; 78.1 \%)$ and 7 as traumatic $(7 / 32 ; 21.9 \%)$. When assessing the $\mathrm{T}^{*}$ values of non fractured vertebrae, patients with low-energy osteoporotic fractures had significantly higher $\mathrm{T}^{*}$ values than those with traumatic fractures $(13.6 \pm 4.3$ vs. $8.4 \pm 2.2 \mathrm{~ms}, \mathrm{P}=0.01)$ (Figures 3,4), significantly lower mean $\mathrm{BMD}$ values $(75.2 \pm 27.7$ vs. $\left.154.3 \pm 33.1 \mathrm{mg} / \mathrm{cm}^{3}, \mathrm{P}<0.001\right)$ as well as a lower mean trabecular number $\left(0.69 \pm 0.08\right.$ vs. $\left.0.93 \pm 0.03 \mathrm{~mm}^{-1}, \mathrm{P}<0.01\right)$, and a significantly greater mean trabecular space $(1.06 \pm 0.16$ vs. $0.56 \pm 0.08 \mathrm{~mm}, \mathrm{P}<0.01$ ); the mean trabecular thickness, however, showed no significant difference between the two subgroups $(0.44 \pm 0.08$ vs. $0.53 \pm 0.09 \mathrm{~mm}, \mathrm{P}=0.11)$ (Table 3). When comparing the mean $\mathrm{T}^{*}$ of the fractured vertebrae, no significant difference could be detected between lowenergy osteoporotic fractures and high-energy traumatic fractures $(12.6 \pm 5.4$ vs. $8.1 \pm 2.4 \mathrm{~ms}, \mathrm{P}=0.10)$.

When evaluating the vertebral $\mathrm{T} 2{ }^{*}$-measurements of non fractured vertebrae in osteoporotic/osteopenic patients and comparing patients with and without vertebral fractures, no significant differences were found between the two 
Table 2 Pearson correlation coefficients calculated for BMD, T2* of non fractured vertebrae, trabecular parameters and age

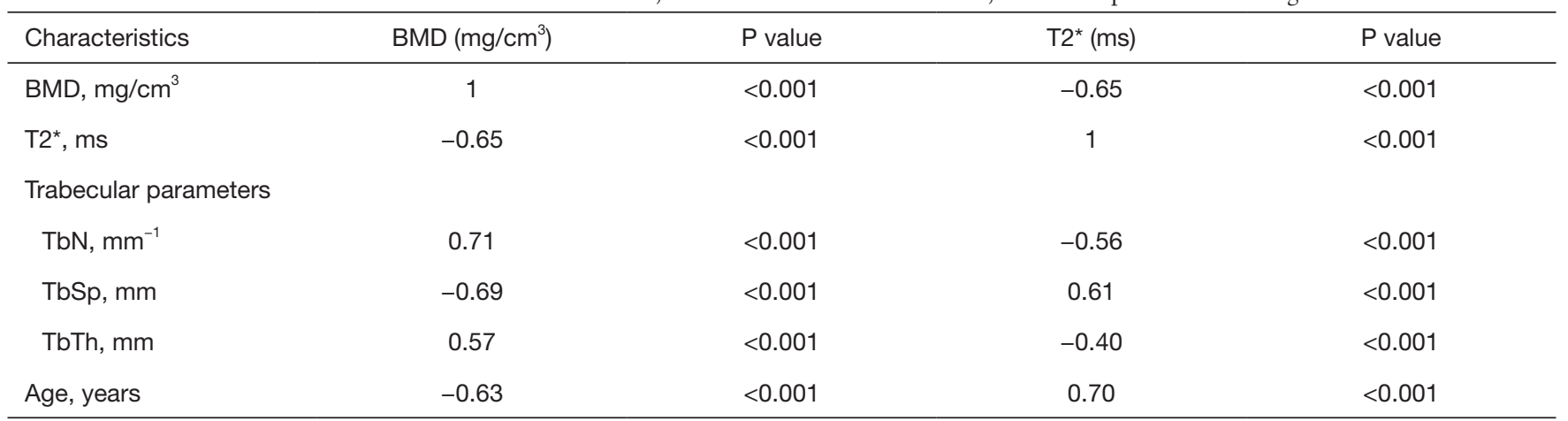

All correlation coefficients are significant at the $\alpha$-level $\mathrm{P}<0.05$. BMD, bone mineral density; TbN, trabecular number; TbSp, trabecular space; TbTh, trabecular thickness.
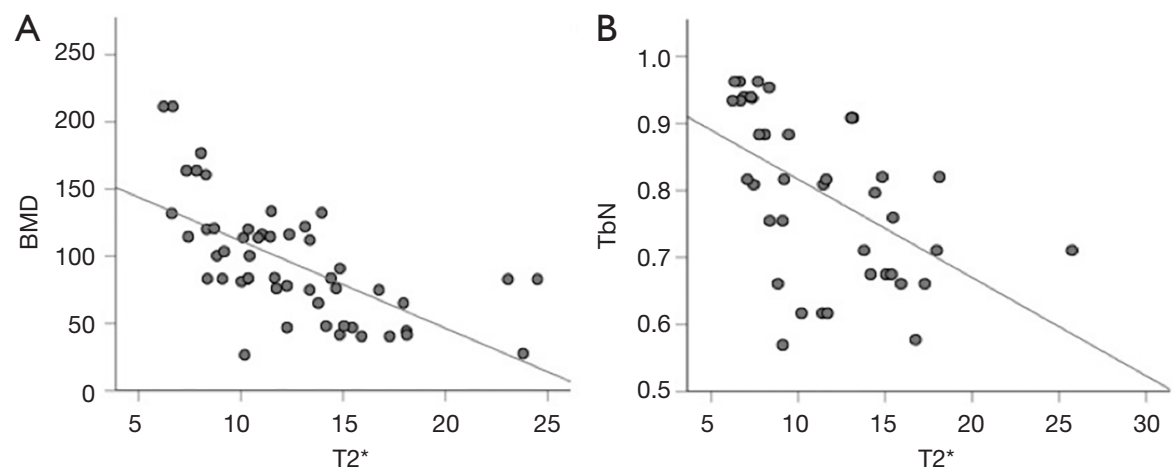

Figure 2 Scatter plot of $\mathrm{T}^{*}$ and $\mathrm{BMD}$ and $\mathrm{TbN}$ respectively. $\mathrm{BMD}$, bone mineral density; TbN, trabecular number.

subgroups ( $13.5 \pm 3.4$ vs. $15.6 \pm 3.5 \mathrm{~ms}, \mathrm{P}=0.40)$. Moreover, there were no significant differences found in mean BMD, mean trabecular space, mean trabecular thickness and mean trabecular number between the two subgroups $(\mathrm{P}>0.05)$.

For the BMD analysis the calculated intra-reader reproducibility RMSE was $6.4 \%$ and for the $\mathrm{T} 2 *$ analysis it was $6.2 \%$.

\section{Discussion}

In this study, we investigated $\mathrm{T} 2{ }^{*}$ mapping using chemical shift encoding-based water-fat separation, its correlation with volumetric trabecular bone mineral density (BMD) and trabecular bone structure parameters and the diagnostic value of the $\mathrm{T} 2{ }^{*}$-measurements for the evaluation of patients with vertebral compression fractures.

$\mathrm{T} 2$ * of vertebral bone marrow correlates with QCT BMD as well as with trabecular structure parameters, allowing the assessment of osteoporosis and the trabecular microstructure. Moreover, T2* values of non-fractured vertebrae in patients with low- and high-energy fractures differ significantly, which enables a radiation-free differentiation between patients with osteoporotic and traumatic vertebral fractures, suggesting its potential as a biomarker for bone fragility. Also, $\mathrm{T} 2 *$ is unable to differentiate between patients with and without fractures in the osteoporotic subcohort.

The analysis of $\mathrm{T}^{*}$ in order to gain information on tissue composition is based on the phenomenon that different types of tissue lead to variations in magnetic susceptibility, which leads to variations in $\mathrm{T} 2 *$ relaxation times. In vertebral bone and bone marrow the $\mathrm{T} 2 *$ measurements are sensitive to inhomogeneities caused by susceptibility differences at the interface of bone marrow and bone trabeculae. These local field inhomogeneities shorten the T2* decay of both the water and fat components, which results in a rapid decay of the measured gradient echo signal with increasing echo time $(25,31)$. It has already 

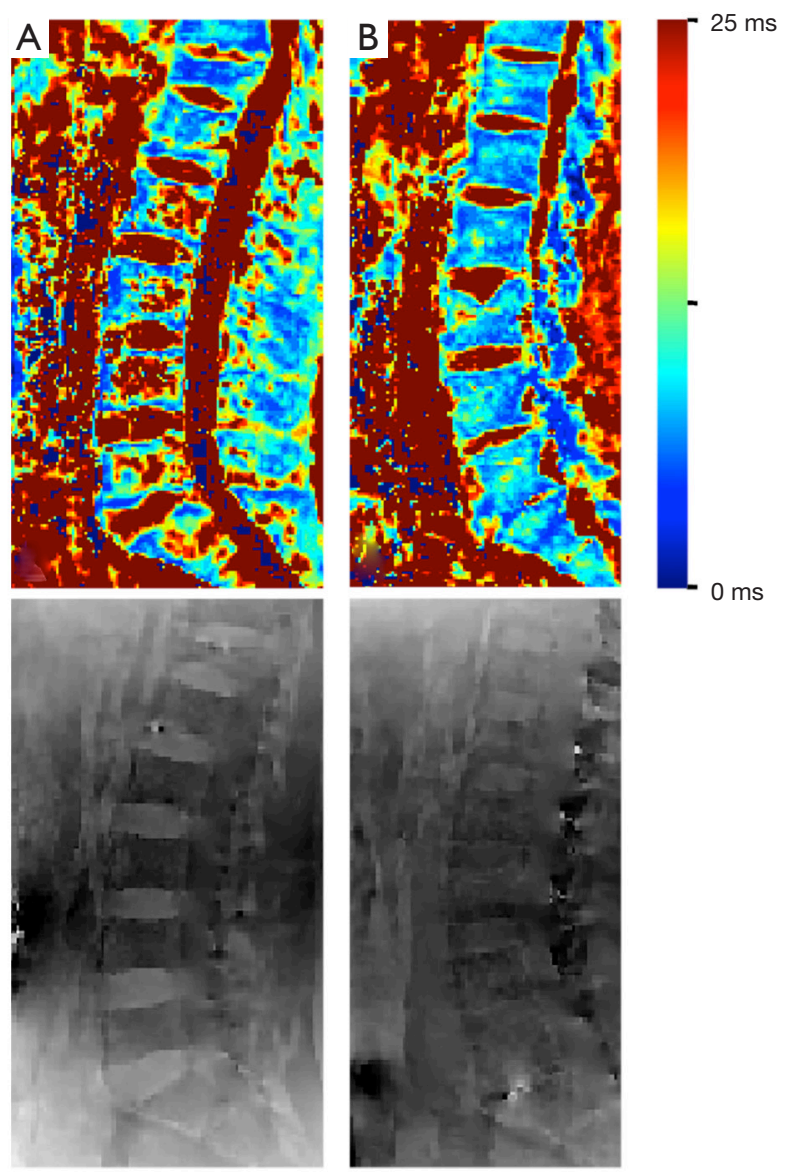

Figure 3 Thoracolumbar spine of a 70 years old female patient with a low energy fracture of Th12 (a and a') and a 49 years old male patient with a high energy fracture of L3 (b and b'). Panels $\mathrm{a}$ and $\mathrm{b}$ show color coded $\mathrm{T} 2{ }^{*}$ maps. The longer $\mathrm{T} 2{ }^{*}$ relaxation times in the non-fractured vertebrae in the patient with the low energy fracture are clearly noticeable. Panels a' and b' show the corresponding B0 field maps.

been shown that $\mathrm{T}^{*}$ of vertebral bone depends on bone density and the microarchitectural trabecular structure, whereas $\mathrm{T}^{*}$ increases with decreasing bone density (11). Previous studies have also demonstrated that MR-based $\mathrm{T} 2{ }^{*}$-measurements enable the differentiation between acute benign and malignant vertebral fractures (17) and that it can be applied to patients with vertebral bone tumors in order to reveal the tumor mass and the invasion of the tumor into the adjacent structures (32).

To our knowledge, it has not been investigated yet whether $\mathrm{T} 2{ }^{*}$ mapping of vertebral bone marrow using chemical shift encoding-based water-fat separation enables

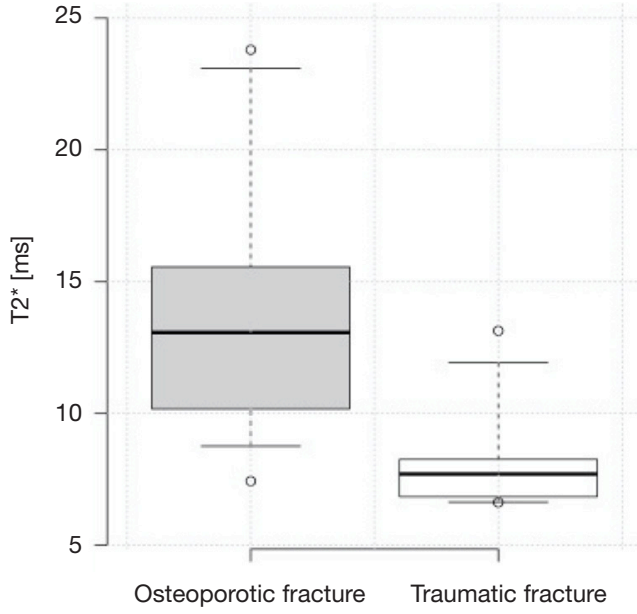

Figure 4 Average T2* of non-fractured lumbar vertebrae in patients with osteoporotic $(n=25)$ and traumatic fractures $(n=7)$.

the further differentiation between patients with highenergy traumatic and low-energy osteoporotic/osteopenic fractures as well as osteoporotic/osteopenic patients with and without vertebral fractures.

In our study, the overall $\mathrm{T} 2{ }^{*}$-measurements of intact vertebrae showed a significant inverse correlation with the bone mineral density which was assessed by using opportunistic QCT measurements. T2* was significantly higher in patients with osteoporosis/osteopenia compared to patients with normal BMD (i.e., $>120 \mathrm{mg} / \mathrm{cm}^{3}$ ). These findings are in line with the previous studies $(10,11)$. As the increase in $\mathrm{T} 2{ }^{*}$ in patients with lower bone mineral density is presumably caused by rarefication of the trabecular bone structure, we investigated the correlation between $\mathrm{T} 2^{*}$ and the bone structure in greater detail; we therefore analysed CT-based trabecular bone parameters ( $\mathrm{TbTh}, \mathrm{TbSp}$, $\mathrm{TbN}$ ) and their correlation with $\mathrm{T} 2 *$. Previous studies demonstrated that $\mathrm{T}^{*}$ correlates with CT-based trabecular bone parameters like trabecular thickness and number $(33,34)$; those studies, however, focused on peripheral skeletal sites (e.g., wrist, calcaneus). To our knowledge, correlation of QCT for microstructure parameters with T2*-measurements using CSE-MRI in vertebral bone marrow has not been demonstrated yet. Our results showed a high and significant correlation between vertebral bone marrow $\mathrm{T}^{*}$ and the trabecular number as well as the trabecular spacing. Overall, $\mathrm{T} 2^{*}$-measurements seem to be a biomarker for the overall bone mineral density as well as the trabecular microarchitecture of the vertebral bone.

In our clinical cohort, $\mathrm{T} 2{ }^{*}$-measurements of non 
Table 3 Subgroup analysis of patients with at least one vertebral fracture

\begin{tabular}{|c|c|c|c|}
\hline Characteristics & High energy fracture $(n=7)$ & Low energy fracture $(n=25)$ & $P$ value \\
\hline $\mathrm{BMD}, \mathrm{mg} / \mathrm{cm}^{3}$ & $154.3 \pm 33.1$ & $75.2 \pm 27.7$ & $<0.01$ \\
\hline \multicolumn{4}{|l|}{$\mathrm{T}^{*}, \mathrm{~ms}$} \\
\hline Non fractured vertebrae & $8.4 \pm 2.2$ & $13.6 \pm 4.3$ & 0.01 \\
\hline \multicolumn{4}{|l|}{ Trabecular parameters } \\
\hline $\mathrm{TbN}, \mathrm{mm}^{-1}$ & $0.93 \pm 0.03$ & $0.69 \pm 0.08$ & $<0.01$ \\
\hline TbSp, mm & $0.56 \pm 0.08$ & $1.06 \pm 0.16$ & $<0.01$ \\
\hline TbTh, mm & $0.53 \pm 0.09$ & $0.44 \pm 0.08$ & 0.11 \\
\hline
\end{tabular}

BMD, bone mineral density; TbN, trabecular number; TbSp, trabecular space; TbTh, trabecular thickness.

fractured vertebrae were able to differentiate between patients with low-energy osteoporotic fractures and patients with traumatic fractures, showing significantly prolonged $\mathrm{T}_{2}$ * in those with low-energy osteoporotic fractures. Accordingly, between patients with osteoporotic and traumatic fractures the trabecular number and trabecular space also showed significant differences.

When measuring $\mathrm{T}^{*}$ in fractured vertebrae, the values are on average very close to the measurements of the non fractured vertebrae (Table 3). However, the T2* values in low-energy VCFs tend to be lower on average with a larger standard deviation $(12.6 \pm 5.4$ vs. $13.6 \pm 4.3 \mathrm{~ms})$. There are several factors in a fractured vertebra that might influence the $\mathrm{T}^{*}$ measurements, e.g., fracture age, edema, fracture morphology and compaction of the vertebra, which in turn might lead to an underestimation of the $\mathrm{T} 2^{*}$ value when trying to assess the general bone structure. As a result, when comparing the mean $\mathrm{T}^{*}$ of the fractured vertebrae, no significant difference could be detected between low-energy osteoporotic fractures and high-energy traumatic fractures in our study population. Our data suggests that the clinical value of $\mathrm{T}^{*}$ measurements using chemical shift encodingbased MRI lies in the evaluation of the whole lumbar spine and the radiation-free estimation of the bone mineral density.

Our data shows that $\mathrm{T}^{*}$-analysis of the vertebral bone using chemical shift encoding-based water-fat separation has a great potential to be a useful biomarker for bone fragility since it can assess the BMD and microstructural architecture of the bone and differentiate between patients with different types of vertebral fractures. This indicates that CSE-MRI-based T2*-analysis of the vertebral spine allows the assessment for osteoporosis and microstructural bone changes, which are usually assessed using radiationdependent techniques such as DXA or dedicated QCT. In a clinical setting MR imaging could therefore not only be used for the assessment of the presence of a fracture and the fracture age, as it is commonly requested, but also in order to gain reliable additional information on the microarchitectural bone structure and bone mineral density as well as the origin of the fracture (e.g., osteoporotic or traumatic).

This study has limitations. The sample sizes, especially for the subanalyses were fairly small. Especially the number of patients with osteoporosis without vertebral fracture as well as the number of patients with traumatic fractures is fairly low. Also, the age difference between patients with high- and low-energy fractures is rather high and is a potential confounder as BMD and $\mathrm{T}^{*}$ correlate with age. Furthermore, our study focuses on cross-sectional data only; it might be of interest how $\mathrm{T}^{*}$ performs as a predictive parameter for fracture risk e.g., in osteoporotic patients that develop osteoporotic fractures during follow-up and how it compares to radiation-dependant established modalities. Overall larger study cohorts and longitudinal study designs are needed in the future.

Two different MRI scanners were used in the study; however, the two scanners were running on identical scanner software releases and our implementation of the pulse sequence design and image reconstruction was identical in the two scanners. There were no significant differences found between the patients scanned at the two 
different scanners regarding patient characteristics. Patients of each subgroup were equally distributed between the two scanners. The off-line water-fat separation processing was centralized and identical for the data originating both systems. We therefore expect minimal effect of the scanner hardware on the measured T2* values. A potential limitation for this method in a clinical setting might be the rather long scan times, which originate from the comparably high isotropic spatial resolution and the consequently need for an interleaved acquisition scheme to achieve a reasonable echo time step (delta TE).

In conclusion, $\mathrm{T} 2^{*}$ mapping of vertebral bone marrow using chemical shift encoding-based water-fat separation allows the assessment of the bone mineral densitiy and osteoporosis as well as the trabecular microstructure and enables a radiation-free differentiation between patients with low-energy osteoporotic and high-energy traumatic vertebral fractures, suggesting its potential as a biomarker for bone fragility.

\section{Acknowledgments}

Funding: None.

\section{Footnote}

Conflicts of Interest: All authors have completed the ICMJE uniform disclosure form (available at http://dx.doi. org/10.21037/qims-20-1373). DCK receives grant support from Philips Healthcare. The other authors have no conflicts of interest to declare.

Ethical Statement: The authors are accountable for all aspects of the work in ensuring that questions related to the accuracy or integrity of any part of the work are appropriately investigated and resolved. The study was conducted in accordance with the Declaration of Helsinki (as revised in 2013). The study was approved by the local institutional review board (Ethics Commission of the Medical Faculty, Technical University of Munich, Germany; Ethics proposal number 70/17S). Written informed consent was obtained from all study participants.

Open Access Statement: This is an Open Access article distributed in accordance with the Creative Commons Attribution-NonCommercial-NoDerivs 4.0 International License (CC BY-NC-ND 4.0), which permits the noncommercial replication and distribution of the article with the strict proviso that no changes or edits are made and the original work is properly cited (including links to both the formal publication through the relevant DOI and the license). See: https://creativecommons.org/licenses/by-nc-nd/4.0/.

\section{References}

1. Sanfélix-Gimeno G, Sanfelix-Genovés J, Hurtado I, Reig-Molla B, Peiró S. Vertebral fracture risk factors in postmenopausal women over 50 in Valencia, Spain. A population-based cross-sectional study. Bone 2013;52:393-9.

2. Consensus development conference: diagnosis, prophylaxis, and treatment of osteoporosis. Am J Med 1993;94:646-50.

3. Melton LJ 3rd. Epidemiology of Spinal Osteoporosis. Spine 1997;22:2S-11S.

4. Hernlund E, Svedbom A, Ivergård M, Compston J, Cooper C, Stenmark J, McCloskey EV, Jönsson B, Kanis JA. Osteoporosis in the European Union: medical management, epidemiology and economic burden. A report prepared in collaboration with the International Osteoporosis Foundation (IOF) and the European Federation of Pharmaceutical Industry Associations (EFPIA). Arch Osteoporos 2013;8:136.

5. Miller PD. Management of severe osteoporosis. Expert Opin Pharmacother 2016;17:473-88.

6. Kanis JA, Svedbom A, Harvey N, McCloskey EV. The osteoporosis treatment gap. J Bone Miner Res 2014;29:1926-8.

7. American College of Radiology (2018). ACR Appropriateness Criteria ${ }^{\circledR}$ Suspected Spine Trauma. Available online: https://acsearchacrorg/docs/69359/ Narrative/

8. Majumdar S, Thomasson D, Shimakawa A, Genant HK. Quantitation of the susceptibility difference between trabecular bone and bone marrow: Experimental studies. Magn Reson Med 1991;22:111-27.

9. Wehrli FW, Ford JC, Attie M, Kressel HY, Kaplan FS. Trabecular structure: preliminary application of MR interferometry. Radiology 1991;179:615-21.

10. Wehrli FW, Song HK, Saha PK, Wright AC. Quantitative MRI for the assessment of bone structure and function. NMR Biomed 2006;19:731-64.

11. Wu HZ, Zhang XF, Han SM, Cao L, Wen JX, Wu WJ, Gao BL. Correlation of bone mineral density with MRI $\mathrm{T} 2 *$ values in quantitative analysis of lumbar osteoporosis. Arch Osteoporos 2020;15:18. 
12. Griffith JF, Yeung DKW, Antonio GE, Lee FKH, Hong AWL, Wong SYS, Lau EMC, Leung PC. Vertebral Bone Mineral Density, Marrow Perfusion, and Fat Content in Healthy Men and Men with Osteoporosis: Dynamic Contrast-enhanced MR Imaging and MR Spectroscopy. Radiology 2005;236:945-51.

13. Karampinos DC, Ruschke S, Dieckmeyer M, Diefenbach M, Franz D, Gersing AS, Krug R, Baum T. Quantitative MRI and spectroscopy of bone marrow. J Magn Reson Imaging 2018;47:332-53.

14. Kühn JP, Hernando D, Meffert PJ, Reeder S, Hosten N, Laqua R, Steveling A, Ender S, Schröder H, Pillich DT. Proton-density fat fraction and simultaneous R2* estimation as an MRI tool for assessment of osteoporosis. Eur Radiol 2013;23:3432-9.

15. Zhao Y, Huang M, Ding J, Zhang X, Spuhler K, Hu S, Li M, Fan W, Chen L, Zhang X, Li S, Zhou Q, Huang C. Prediction of Abnormal Bone Density and Osteoporosis From Lumbar Spine MR Using Modified Dixon Quant in 257 Subjects With Quantitative Computed Tomography as Reference. J Magn Reson Imaging 2019;49:390-9.

16. Li GW, Xu Z, Chen QW, Tian YN, Wang XY, Zhou L, Chang SX. Quantitative evaluation of vertebral marrow adipose tissue in postmenopausal female using MRI chemical shift-based water-fat separation. Clin Radiol 2014;69:254-62.

17. Schmeel FC, Luetkens JA, Feißt A, Enkirch SJ, Endler CHJ, Wagenhäuser PJ, Schmeel LC, Träber F, Schild HH, Kukuk GM. Quantitative evaluation of T2* relaxation times for the differentiation of acute benign and malignant vertebral body fractures. Eur J Radiol 2018;108:59-65.

18. Diamantopoulos AP, Rohde G, Johnsrud I, Skoie IM, Hochberg M, Haugeberg G. The epidemiology of lowand high-energy distal radius fracture in middle-aged and elderly men and women in Southern Norway. PloS one 2012;7:e43367.

19. Baum T, Müller D, Dobritz M, Rummeny EJ, Link TM, Bauer JS. BMD measurements of the spine derived from sagittal reformations of contrast-enhanced MDCT without dedicated software. Eur J Radiol 2011;80:e140-5.

20. Löffler MT, Jacob A, Valentinitsch A, Rienmüller A, Zimmer C, Ryang YM, Baum T, Kirschke JS. Improved prediction of incident vertebral fractures using opportunistic QCT compared to DXA. Eur Radiol 2019;29:4980-9.

21. Roski F, Hammel J, Mei K, Baum T, Kirschke JS, Laugerette A, Kopp FK, Bodden J, Pfeiffer D, Pfeiffer F, Rummeny EJ, Noël PB, Gersing AS, Schwaiger BJ.
Bone mineral density measurements derived from duallayer spectral CT enable opportunistic screening for osteoporosis. Eur Radiol 2019;29:6355-63.

22. American College of Radiology (2018). ACR-SPR-SSR practice parameter for the performance of musculoskeletal quantitative computed tomography (QCT). Available online: https://wwwacrorg/-/media/ACR/Files/PracticeParameters/qctpdf?la=en

23. Ruschke S, Eggers H, Kooijman H, Diefenbach MN, Baum T, Haase A, Rummeny EJ, Hu HH, Karampinos DC. Correction of phase errors in quantitative water-fat imaging using a monopolar time-interleaved multi-echo gradient echo sequence. Magn Reson Med 2017;78:984-96.

24. Karampinos DC, Yu H, Shimakawa A, Link TM, Majumdar S. T1-corrected fat quantification using chemical shift-based water/fat separation: Application to skeletal muscle. Magn Reson Med 2011;66:1312-26.

25. Karampinos DC, Ruschke S, Dieckmeyer M, Eggers H, Kooijman H, Rummeny EJ, Bauer JS, Baum T. Modeling of T2* decay in vertebral bone marrow fat quantification. NMR Biomed 2015;28:1535-42.

26. Ren J, Dimitrov I, Sherry AD, Malloy CR. Composition of adipose tissue and marrow fat in humans by $1 \mathrm{H}$ NMR at 7 Tesla. J Lipid Res 2008;49:2055-62.

27. Boehm C, Diefenbach MN, Makowski MR, Karampinos DC. Improved body quantitative susceptibility mapping by using a variable-layer single-min-cut graph-cut for fieldmapping. Magn Reson Med 2021;85:1697-712.

28. Diefenbach MN, Liu C, Karampinos DC. Generalized parameter estimation in multi-echo gradient-echo-based chemical species separation. Quant Imaging Med Surg 2020;10:554-67.

29. Bauer JS, Kohlmann S, Eckstein F, Mueller D, Lochmüller EM, Link TM. Structural Analysis of Trabecular Bone of the Proximal Femur Using Multislice Computed Tomography: A Comparison with Dual X-Ray Absorptiometry for Predicting Biomechanical Strength In Vitro. Calcif Tissue Int 2006;78:78-89.

30. Bauer JS, Link TM, Burghardt A, Henning TD, Mueller D, Majumdar S, Prevrhal S. Analysis of Trabecular Bone Structure with Multidetector Spiral Computed Tomography in a Simulated Soft-Tissue Environment. Calcif Tissue Int 2007;80:366-73.

31. Chavhan GB, Babyn PS, Thomas B, Shroff MM, Haacke EM. Principles, Techniques, and Applications of T2*-based MR Imaging and Its Special Applications. Radiographics 2009;29:1433-49.

32. Tang MY, Chen TW, Zhang XM, Huang XH. GRE T2*- 
Weighted MRI: Principles and Clinical Applications.

Biomed Res Int 2014;2014:312142.

33. Wurnig MC, Calcagni M, Kenkel D, Vich M, Weiger M, Andreisek G, Wehrli FW, Boss A. Characterization of trabecular bone density with ultra-short echo-time MRI at 1.5, 3.0 and 7.0 T - comparison with micro-computed

Cite this article as: Leonhardt Y, Gassert FT, Feuerriegel G, Gassert FG, Kronthaler S, Boehm C, Kufner A, Ruschke S, Baum T, Schwaiger BJ, Makowski MR, Karampinos DC, Gersing AS. Vertebral bone marrow T2* mapping using chemical shift encoding-based water-fat separation in the quantitative analysis of lumbar osteoporosis and osteoporotic fractures. Quant Imaging Med Surg 2021;11(8):3715-3725. doi: 10.21037/qims-20-1373 tomography. NMR Biomed 2014;27:1159-66.

34. Rebuzzi M, Vinicola V, Taggi F, Sabatini U, Wehrli FW, Capuani S. Potential diagnostic role of the MRI-derived internal magnetic field gradient in calcaneus cancellous bone for evaluating postmenopausal osteoporosis at $3 \mathrm{~T}$. Bone 2013;57:155-63. 